\title{
Analysis of imperfections in a micromachined tunable-cavity interferometer
}

\author{
Jung-sik Moon and Andrei M. Shkel \\ Microsystems Laboratory, Department of Mechanical and Aerospace Engineering, \\ University of California, Irvine, CA, USA
}

\begin{abstract}
An alternative to a classical wavelength interferometer (an array of hand-assembled etalons consisting of two semitransparent mirrors separated by a fixed-cavity) is the implementation of wide band tunable filter using Micro-ElectroMechanical Systems (MEMS) technology. This approach will allow a single tunable device to replace an array of fixed-cavity filters reducing cost and parts. MEMS technology offers many advantages, including scalability for wide tuning range, sensitivity for precision sensing, and batch fabrication capability for cost reduction. While at the same time, MEMS technology introduces many new challenges, which include fabrication yield, device reproducibility, and fabrication imperfections - all are factors seriously limiting performance of MEMS interferometers. Without defects, reflectivity must be greater than $99.69 \%$ in order to achieve finesse of 1000 to be useful for DWDM applications. Though, the presence of defects limits performance and becomes more pronounced at higher reflectivity values. For example, component misregistration while fabricating MEMS interferometer with $95 \%$ and $98 \%$ reflectivity values, result in the reduction of effective finesse of $10 \%$ and $42 \%$, respectively. This paper discusses several models for analyzing imperfections in MEMS tunable-cavity interferometers. Based on thermal expansion and component misregistration analysis, we conclude that a passive MEMS-based filter cannot achieve performance required for DWDM applications.
\end{abstract}

Keywords: Fabry-Perot Interferometer, Etalon, Finite Element Analysis, Multiphysics modeling, MEMS

\section{INTRODUCTION}

Classical wavelength interferometers are a collection of hand assembled etalons, consisting of two semi-transparent mirrors separated by a fixed-cavity. Individual etalons are designed with a different cavity size, each responsible for filtering a specific wavelength. Commonly used in telescopes and optical measurement devices, tunable filters are in high demand in the telecommunication industry, where the goal is to filter wavelengths from $1550 \mathrm{~nm}$ to $1630 \mathrm{~nm}$, crucial for Dense Wavelength Division Multiplexing (DWDM). Typically, DWDM requires a wavelength interferometer to filter forty or more channels/wavelengths. Therefore assembling forty or more discrete etalons (approximately $\$ 300 /$ ea) to achieve a wide tunable range needed for DWDM technology can be quite costly.

An alternative to this discrete approach is the implementation of wide band tunable filter using MEMS technology, in a single device. This approach will allow a single tunable device to replace an array of fixed-cavity filters. MEMS technology offers many advantages, including scalability for wide tuning range, sensitivity for precision sensing, and batch fabrication capability for cost reduction. However, MEMS technology introduces many new challenges, such as fabrication yield, device reproducibility, and fabrication imperfections, all are factors limiting performance. Although high sensitivity may be beneficial for precision sensors, it is equally disadvantageous due to sensitivity to undesirable perturbations. Considering all advantages and disadvantages, it is critical to understand how limitations inherent to MEMS technology affect performance of a micro-machined tunable-cavity filter.

Four possible tunable filters are presented in Fig. 1, they include diffraction grating filter, tunable cavity interferometer, variable angle entail, and "linear sliding" Fabry-Perot interferometer. Diffraction grating filter induces interference through the grating surface, and tuning is achieved by rotation (changing the incident beam angle). Tunable cavity interferometer is a Fabry-Perot interferometer (FPI), where incident beam enters the cavity normal

Further author information: (Send correspondence to J.M.)

J.M.: E-mail: jmoon@uci.edu

A.S.: E-mail: ashkel@uci.edu

Web: http://mems.eng.uci.edu 
to the mirrors, and a light wave of selected frequency resonates in the cavity through interference. The resonating cavity length equals multiples of half wavelength, and tuning is achieved by changing the cavity length. Similarly, the variable angle etalon is a Fabry-Perot interferometer, but with a fixed cavity. Rather than varying the cavity length, tuning is achieved by changing the incident beam angle. The resonating condition for an etalon is met when the cavity length equals multiples of half wavelength divided by cosine of the angle measured from the mirror normal, Fig. 2. Finally, the "linear sliding" filter operates like the tunable cavity filter, but the cavity length is varied by sliding the tapered surface.

\begin{tabular}{|c|c|c|c|}
\hline Diffraction Grating & Tunable Cavity & Rotating Etalon & "Linear Sliding" Tuning \\
\hline Rotation & Variable & Light Output & Input \\
\hline
\end{tabular}

Figure 1. Four possible tuning methods for interferometry.

Diffraction grating filter is capable of having a wide tunable range, but high polarization is a problem. Likewise, rotating etalon has high polarization dependence and characteristics change as a result of changing incident beam angle, while tuning. Although, stability and reliability of a rotational etalon filter is good, it has limited tuning range. "Linear sliding" filter is stable/reliable, has low polarization dependence loss, and has constant characteristics over the whole tuning range. However, as a disadvantage, special fabrication techniques are needed in order to generate the tapered surface. While exploring available tuning methods suitable for micro-machining technology, wide tuning capability, low polarization, and reduced processing steps made tunable-cavity FPF most attractive. Therefore, a tunable-cavity Fabry-Perot filter is the focus of this paper.

In Sec. 2, principle of operation and performance of an ideal Fabry-Perot interferometer is presented. Based on principles of Sec. 2, we will explain why FPF is a versatile device capable of many functions. In Sec. 3, methodology for developing relationship between mirror imperfection and performance is covered. Sec. 4 presents results of modeling performance limits caused by component misregistration and thermal expansion, and using these results we conclude in Sec. 5 by stating limitations of a passive MEMS tunable cavity Fabry-Perot filter and offer suggestions at improving performance.

\section{FABRY-PEROT PRINCIPLE}

A Fabry-Perot filter (FPF) is a device that transmits a selected wavelength or frequency by interference of multiple beams through a reflective cavity formed by two flat, partially transmitting, parallel mirrors separated by a medium, Fig. 2.

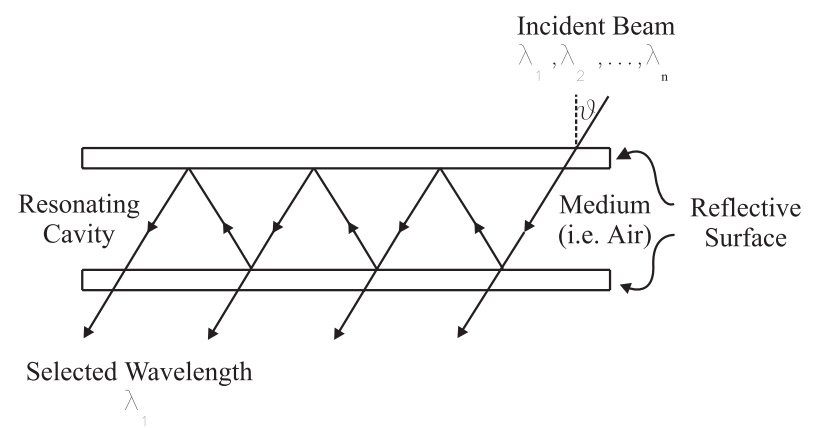

Figure 2. Operation of a Fabry-Perot interferometer. 
Incident light enters the Fabry-Perot cavity, and undergoes multiple reflections, where individual wavelengths interfere constructively or destructively. Wavelengths in phase interfere constructively and wavelengths out of phase interfere destructively. The constructive wavelength resonates, and this condition is satisfied when the following expression holds ${ }^{1}$ :

$$
n d \cos \theta=\frac{m \lambda}{2}
$$

where $\theta$ is the incident light angle normal to the mirror, $\lambda$ is wavelength, $d$ is the cavity length, $n$ is the refractive index of the medium, and $m$ is the fringe order number. For normal incident light, with air as the medium $(n=1)$, the resonating cavity equals multiples of a half wavelength. Resonating light is analogous to a mechanical system operating on the resonance principle. When the resonance conditions are met, a selected portion of the light spectrum resonates and escapes the Fabry-Perot cavity. An important equation describing the intensity $(T)$ of the transmitted wavelength for an ideal FPF is given by the Airy function ${ }^{1}$ :

$$
T=\left(1-\frac{A}{(1-R)}\right)^{2} \frac{1}{1+\frac{4 R \sin ^{2}\left(k d \cos \left(\theta-\frac{\pi}{2}\right)\right)}{(1-R)^{2}}}
$$

where $A$ is mirror absorption, $T$ is mirror transmittance, $R$ is mirror reflectivity, and $k$ equals to $2 \pi / \lambda$. From examination of Eq. (2), intensity is influenced by cavity gap, incident beam angle, and the refractive index. By taking advantage of these parameters, high precision sensing or light modulating applications are realized.

\subsection{Finesse}

Finesse is a figure of merit for defining the performance of a Fabry-Perot filter, determining the number of channels or fringes it can transmit effectively. A high finesse value results in sharper transmission peaks and narrow bandwidth, increasing the resolution and allowing additional channels for greater data density. Therefore, understanding factors affecting finesse of a filter is crucial in defining the performance limits.

Wavelength between consecutive interference fringes is called the free spectral range $\left(F S R_{R}\right)$, given by

$$
F S R_{R}=\frac{\lambda^{2}}{2 d n}
$$

and full width of the transmittance curve at half of the maximum intensity is called the full width half maximum $(F W H M)$, given by

$$
F W H M_{R}=\frac{\lambda(1-R)}{n \pi \sqrt{R}},
$$

then reflectivity finesse $\left(F_{R}\right)$ is defined as the ratio of the free spectral range over the full width half maximum ${ }^{1}$ :

$$
F_{R}=\frac{\pi \sqrt{R}}{(1-R)}
$$

Reflectivity finesse $\left(F_{R}\right)$ is only dependent on the reflectivity of the mirrors, assuming that both mirrors have equal reflectivity. Intuitively, increasing the reflectivity increases the finesse. However, it will be shown in Sec. 3 that mirror defects limit practical values of reflectivity. Fig. 3 is an illustration of an interferometer's transmission profile through several fringe orders or transmission peaks with notations for defining performance. An ideal Fabry-Perot interferometer has sharp transmission peaks, and small bandwidth, whereas the non-ideal Fabry-Perot interferometer has wider bandwidth, reducing the finesse. 


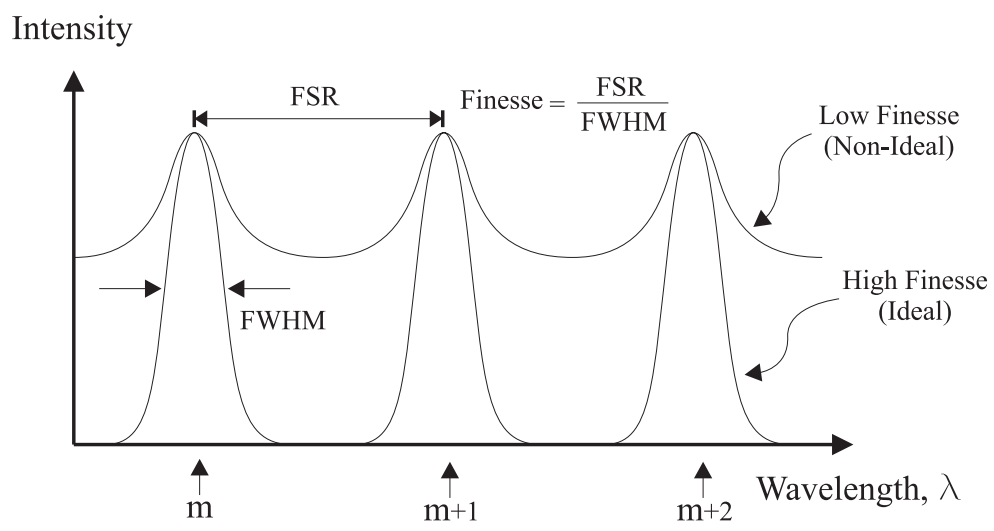

Figure 3. Interferometer's transmission profile and notions defining device performance.

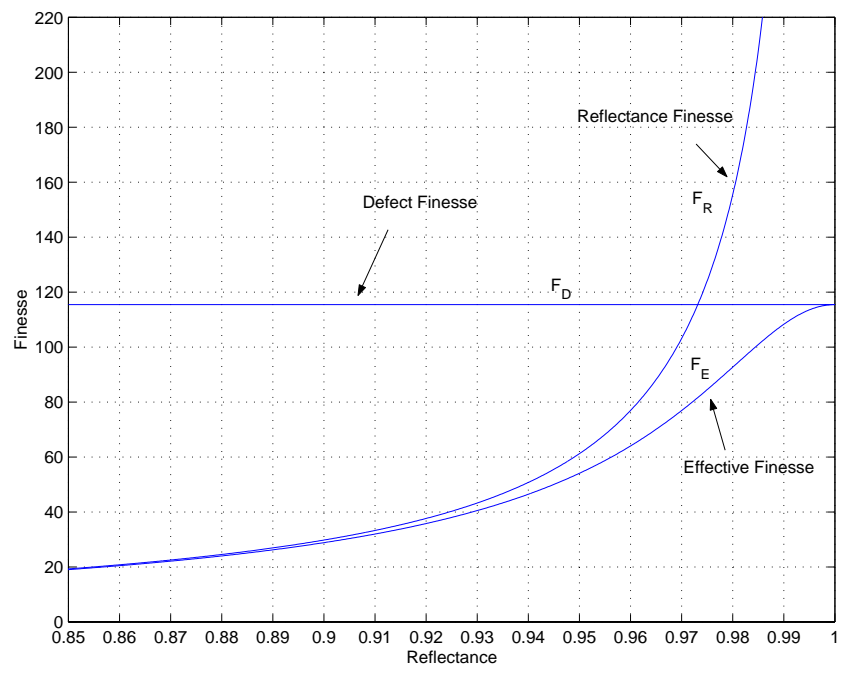

Figure 4. Reflectivity finesse defines performance of a Fabry-Perot interferometer, under ideal conditions (i.e. Perfectly flat mirrors). In practice a Fabry-Perot interferometer is non-ideal, and it's performance is defined by the effective finesse, where it asymptotically reaches a limit determined by the defect finesse.

\section{METHODOLOGY}

In practice, effective finesse $\left(F_{E}\right)$ of a Fabry-Perot filter is measured empirically, and reflectivity finesse is calculated from Eq. (5). Using these results, the defect finesse $\left(F_{D}\right)$ is deduced from the following equation [3]:

$$
\frac{1}{F_{E}^{2}}=\frac{1}{F_{R}^{2}}+\frac{1}{F_{D}^{2}}
$$

Fig. 4 is an example illustrating defect finesse as the limiting condition for achieving high finesse. The defect in this example assumes parallelism error $\left(\delta_{p}\right)$ of $20 \mathrm{~nm}$.

A good survey for calculation of effective finesse is given by Palik. ${ }^{2}$ The real Fabry-Perot filter's performance is limited by the mirror's imperfections and reflectivity. One common approach for modeling defects is by projecting all surface defects to one reflective surface, considering the other mirror perfectly flat, and convoluting the surface aberrations and the Airy function, Eq. (2). This technique assumes that the non-ideal mirror is a collection of infinitesimal perfectly parallel mirrors with variable cavity lengths, and the integration over elemental mirrors results in the transmission profile of the non-ideal surface. ${ }^{3}$ Three distinct types of defects are used to model plate defects and are illustrated in Fig. 5(a), which is discussed by Atherton. ${ }^{4} \delta_{p}$ is the circular aperture (parabolic error), $\delta_{s}$ is the square aperture (parallelism error), and $\delta_{r m s}$ is the surface irregularities following the Gaussian distribution 
surface (defects are measured from the planar reference). An illustration of the convolution of a parallelism error can be seen in Fig. 5(b). It is important to remember that individual defects are difficult to measure empirically, therefore the contribution of individual defects are not known.

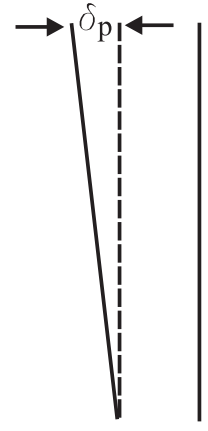

Parallel Deviation

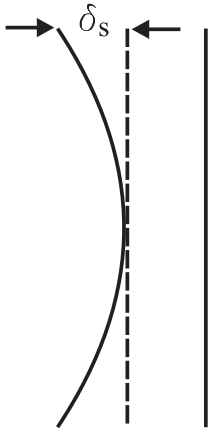

Spherical Deviation

(a)

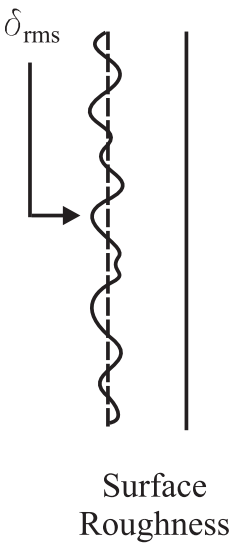

Rurface

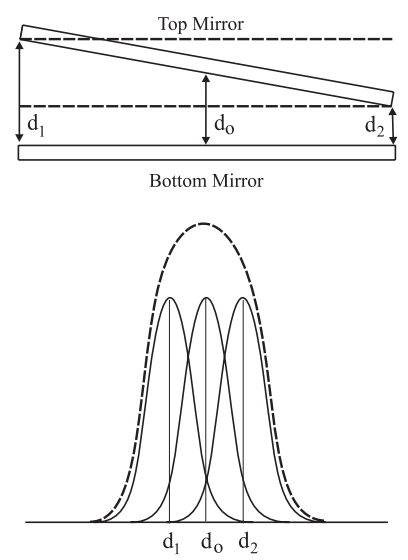

(b)

Figure 5. (a)Classification of plate defects (adopted from Atherton). (b)Parallelism error model.

Derived from results, ${ }^{4}$ defect finesse due to departure from parallelism, spherically bowed plates, and surface irregularities is given by

$$
\begin{aligned}
F_{p} & =\frac{\lambda}{3^{\frac{1}{2}} \delta_{p}} \\
F_{s} & =\frac{\lambda}{2 \delta_{s}} \\
F_{r m s} & =\frac{\lambda}{4.7 \delta_{r m s}}
\end{aligned}
$$

where $\delta_{p}$ and $\delta_{s}$ are the parallel and spherical deviations from a planar reference, and $\delta_{r m s}$ is the root-mean-square deviation following a Gaussian distribution. Representing all three defects simultaneously yields ${ }^{5}$

$$
F_{D}=\left[\frac{1}{F_{p}^{2}}+\frac{1}{F_{s}^{2}}+\frac{1}{F_{r m s}^{2}}\right]^{-\frac{1}{2}}=\frac{\lambda}{\left(3 \delta_{p}^{2}+4 \delta_{s}^{2}+22 \delta_{r m s}^{2}\right)^{\frac{1}{2}}}
$$

Once $F_{D}$ is known, $F_{E}$ is calculated by substituting Eq. (7) into Eq. (6). Generally, a good approximation of $F_{E}$ is made with the assumption that imperfect mirrors have defects which are Lorentzian, and $F_{D}>F_{R}$. Analogous to Eq. (5), the notion of effective reflectivity $R_{E}$ is given by

$$
F_{E}=\frac{\pi \sqrt{R_{E}}}{1-R_{E}}
$$

Further, the transmission function $T(\lambda)$ of an imperfect etalon can be written as

$$
T(\lambda)=T_{p k}\left[\frac{\left(1-R_{E}\right)^{2}}{1+R_{E}^{2}-2 R_{E} \cos (2 \pi m)}\right]
$$

where the transmission peak $T_{p k}$ is

$$
T_{p k}=\left(1-\frac{A}{1-R}\right)^{2}\left(\frac{1-R}{1+R}\right)\left(\frac{1+R_{E}}{1-R_{E}}\right)
$$




\section{PERFORMANCE LIMITS}

Using results described in Sec. 3, a relationship between mirror imperfections and effective finesse is developed. In particular, defects arising as a result of mask misregistration and thermal expansion are modeled. Results of modeling are projected to parallel deviations from a planar surface, and then used to solve for effective finesse Eq. (6) and the transmission profile, Eq. (9). Although, in a real system, all imperfections are present simultaneously, the models serve as an illustration to performance limitations caused by common problems related to MEMS technology.

\subsection{Coupled-Field Modeling}

ANSYS Multiphysics finite element modeling package was used to solve for the coupled electrostatic/structural system response using the command macro ESSOLV. The solid model of the device included a $100 \mu \mathrm{m} \times 100 \mu \mathrm{m}$ mirror suspended by four beams ( $8 \mu \mathrm{m}$ wide, $2 \mu \mathrm{m}$ thick, $60 \mu \mathrm{m}$ long) on four corners and a $100 \mu m \times 100 \mu m$ electrode placed under the suspended mirror, separated by an air filled $2 \mu \mathrm{m}$ optical cavity. Thermal expansion modeling was done by applying a temperature load on all areas with load steps from $258.15 \mathrm{~K}$ to $358.15 \mathrm{~K}$, in $20 \mathrm{~K}$ increments. Material properties used in simulation were as follows: Young Modulus $=1.7 \times 10^{5}[M P a]$, Poisson's ratio $=0.3$, free space permittivity $=8.85 \times 10^{-6}[\mathrm{pF} / \mu \mathrm{m}]$, electrical permittivity of air $=1[\mathrm{pF} / \mu \mathrm{m}]$, and coefficient of thermal expansion $=2.3 \times 10^{-6}[1 / K]$.

\subsection{Misregistration}

Common defect associated with MEMS is composite misregistration, such as accumulative mask misregistration during micro-machining or during wafer assembly (Fig. 6). Hence, modeling for a tunable-cavity Fabry-Perot filter with

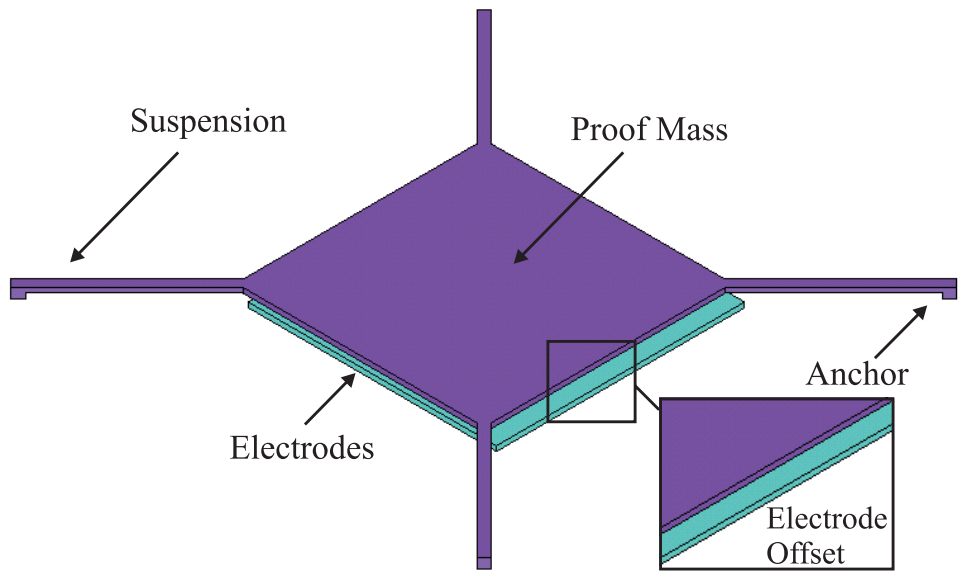

Figure 6. Electrode misalignment example.

expected mask or wafer assembly misregistration was performed. Simulation was done by offsetting the electrodes from $1 \mu \mathrm{m}$ to $4 \mu \mathrm{m}$ along one of the plane axis, in increments of $1 \mu \mathrm{m}$. For each offset parameter, the mirror was actuated by applying $0 \mathrm{~V}$ to $38 \mathrm{~V}$, causing vertical and angular mirror deflection due to uneven electrostatic pressure. When $0.5 \mu \mathrm{m}$ vertical deflection was reached for different offset parameters, angular deflection of 2.5, 3.8, 6.1, and $8.8 \times 10^{-3}$ degrees were calculated, that corresponded to $1 \mu \mathrm{m}, 2 \mu \mathrm{m}, 3 \mu \mathrm{m}$, and $4 \mu \mathrm{m}$ electrode offsets. Fig. 7(a) is a plot of effective finesse as a function of reflectivity for misregistrations $1 \mu m\left(A_{1}\right), 2 \mu m\left(A_{2}\right), 3 \mu m\left(A_{3}\right)$, and $4 \mu \mathrm{m}\left(A_{4}\right)$.

Although the non-parallelism defect is not critical when reflectivity is between $85 \%$ to $93 \%$, finesse dependence becomes much more pronounced as reflectivity increases, Fig. 7(a). For instance, effective finesse of an ideal FPF is 61 and 155 for reflectivity values of $95 \%$ and $98 \%$, but with $4 \mu \mathrm{m}$ mask misregistration causing $8.8 \times 10^{-3}$ degrees tilt $\left(A_{4}\right)$, the effective finesse reduces to 55 and 90 , respectively. This indicates that defects are the limiting factor as reflectivity approaches $100 \%$. A complementing transmission profile for all misregistration at $98 \%$ reflectivity is plotted in Fig. 7(b). As misregistration increases, fringe broadening effect becomes more apparent. 


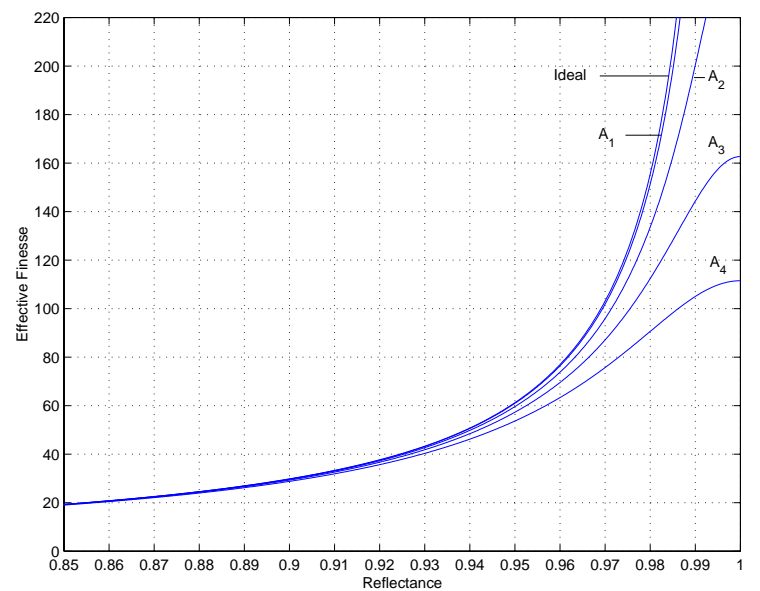

(a)

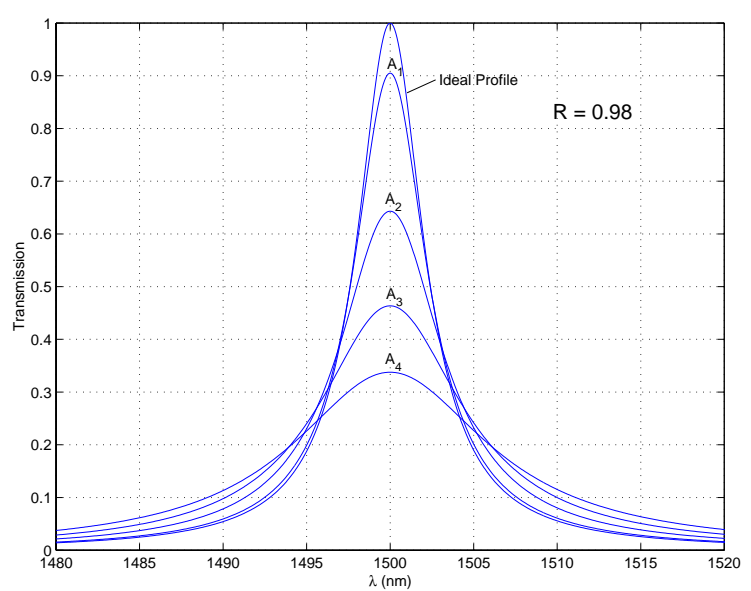

(b)

Figure 7. (a)A reduction in effective finesse as a result of even electrostatic pressure on the suspended mirror caused by component misregistration. (b)Fringe broadening effect as a result of even electrostatic pressure on the mirror caused by component misregistration.

\subsection{Thermal Expansion}

Expected operational temperature range for Fabry-Perot filters is between $-20{ }^{\circ} \mathrm{C}$ and $80{ }^{\circ} \mathrm{C}(258.15 \mathrm{~K}$ to $358.15 \mathrm{~K})$. From thermal expansion modeling results, mirror vertical deflection of $-15.6 \mathrm{~nm},-7.8 \mathrm{~nm}, 0 \mathrm{~nm}, 7.8 \mathrm{~nm}, 15.6 \mathrm{~nm}$, and $23.3 \mathrm{~nm}$ were calculated, corresponding to $258.15 K\left(B_{1}\right), 278.15 K\left(B_{2}\right), 298.15 K\left(B_{3}\right), 313.15 K\left(B_{4}\right), 333.15 K$ $\left(B_{5}\right)$, and $358.15 K\left(B_{6}\right)$ atmospheric temperature, respectively. Similar to the non-parallel mirror case, cavity gap fluctuates with temperature, broadening bandwidth, and reducing finesse. Although temperature fluctuation is not a mirror defect, conceptually one can visualize the thermal expansion as an instantaneous plate deflection. Therefore, thermal expansion of the mirror can be represented as a non-parallel plate defect. Fig. 8(a) is a plot of effective finesse as a function of reflectivity for various thermal expansion cases. Using reflectivity of $95 \%$ and $98 \%$ as in the previous example, $80 \mathrm{~K}$ increase $\left(B_{6}\right)$ decreases effective finesse by approximately $16 \%$ and $47 \%$, respectively. Fringe broadening effects caused by temperature fluctuations are plotted in Fig. 8(b). Initially at $98 \%$ reflectivity, an ideal Fabry-Perot filter's $\left(B_{3}\right)$ bandwidth (full width half maximum) equals $13 \mathrm{~nm}$, at $358.15 \mathrm{~K}\left(B_{6}\right)$ fringe broadens to approximately $42 \mathrm{~nm}$.

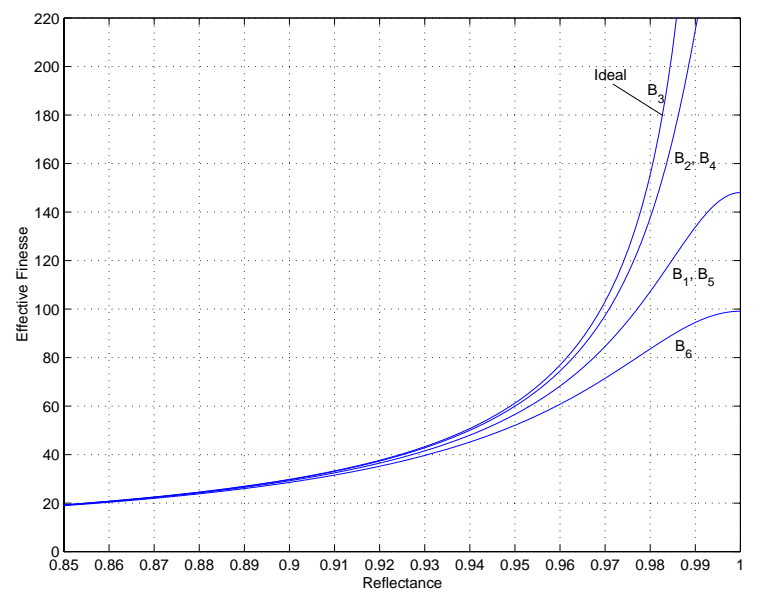

(a)

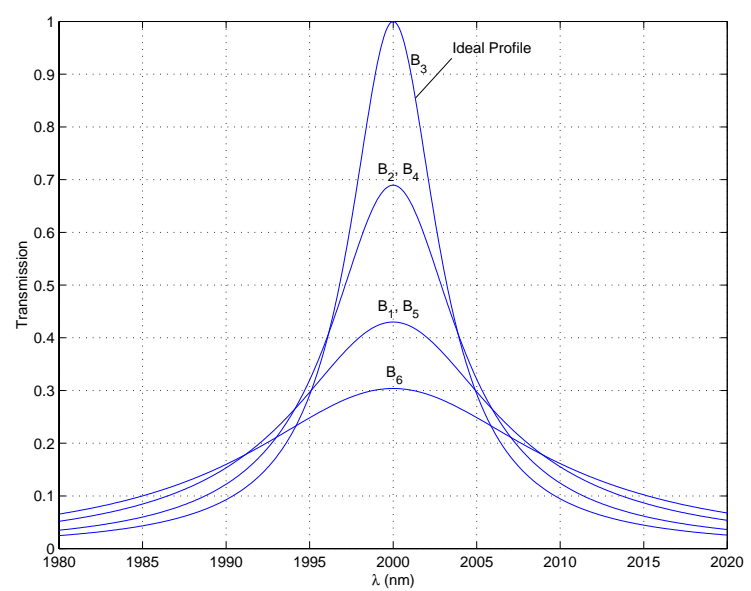

(b)

Figure 8. (a)A reduction in effective finesse caused by thermal expansion. (b)Fringe broadening effect as a result of thermal expansion. 


\section{CONCLUSION}

The paper studied the potential for implementing a high performance tunable-cavity interferometer using MEMS technology. It was concluded, based on thermal expansion and component misregistration analysis, that a passive Fabry-Perot filter cannot achieve performance required for DWDM applications. For this application, in order to transmit 40-channels, finesse must be equal to approximately 1000, where spacing of $100 \mathrm{GHz}(0.8 \mathrm{~nm})$ and bandwidth up to $10 \mathrm{GHz}(0.08 \mathrm{~nm})$ are needed. By depositing gold or silver, reflective materials typically used in optical MEMS applications, $95 \%$ reflectivity can be achieved. At this reflectivity, finesse for an ideal Fabry Perot filter is approximately 61 . However, finesse is further aggravated by misregistration, resulting in mirror tilt on the order of 0.01 degrees, reducing effective finesse by as much as $10 \%$ at $95 \%$ mirror reflectivity. Using the same 40 -channels example, reflectivity must be higher than $99.69 \%$ to be useful for DWDM applications. Current MEMS technology does not satisfy these requirements. However, if MEMS technology is integrated with dielectric coating technology, where multiple dielectric coatings are applied, desirable reflection can be achieved. The use of dielectric coatings requires additional fabrication steps and introduces residual stress that induces mirror curvature. Furthermore, if the defect finesse is greater than the reflectivity finesse, there is no benefit in applying a high reflectivity coating. This tradeoff between development of new fabrication technology and potential for active compensation of imperfections will be the future focus of this research group.

\section{ACKNOWLEDGMENTS}

The authors would like to thank Solus Micro-Technologies Inc. for partial financial support of this project.

\section{REFERENCES}

1. J. Jerman, D. Clift, and S. Mallinson, "Minature Fabry-Perot Interferometer with a Corrugated Silicon Diaphram Support," Sensors and Actuators A (Physical) 29, pp. 140-44, 1990.

2. E. Palik, H. Boukari, and R. Gammon, "Experimental study of the effect of surface defects on the finesse and contrast of a Fabry-Perot interferometer," Applied Optics 35, pp. 38-50, 1996.

3. C. Roychoudhuri and M. Hercher, "Stable multipass Fabry-Perot interferometer: design and analysis," Applied Optics 16, pp. 2514-20, 1977.

4. P. Atherton, N. Reay, J. Ring, and T. Hicks, "Tunable Fabry-Perot Filters," Optical Engineering 20, pp. 806-14, 1981.

5. J. McKay, "Single and tandem Fabry-Perot etalons as solar background filters for lidar," Applied Optics 38, pp 5851-57, 1999. 